\title{
New results and perspectives in neutrino physics
}

\author{
Yury Kudenko ${ }^{1,2,3, *}$ \\ ${ }^{1}$ Institute for Nuclear Research of RAS, 60 October Revolution Pr.7A, 117312 Moscow, Russia \\ ${ }^{2}$ Moscow Institute of Physics and Technology, Institutskiy per. 9, Dolgoprudny, Moscow Region, \\ 141701 Russia \\ ${ }^{3}$ Moscow Engineering Physics Institute, Kashirskoe shosse 31, Moscow, 115409 Russia
}

\begin{abstract}
A brief review of new results and perspectives in neutrino physics is presented. An emphasis on a search for $\mathrm{CP}$ violation in neutrino oscillations and a search for sterile neutrinos is given. Status of measurement of the direct neutrino mass measurement and searches for neutrinoless double beta decay are also discussed.
\end{abstract}

\section{Introduction}

The discovery of neutrino oscillations $[1,2]$ that require neutrinos to be massive provided the convincing evidence of the existence of physics beyond the Standard Model. The neutrino mixing angles $\theta_{12}, \theta_{23}$ and squared-mass differences $\Delta m_{21}^{2}$ and $\left|\Delta m_{32}^{2}\right|$ were measured in experiments with solar, atmospheric, reactor and accelerator neutrinos. Over the past few years, exciting results of observation of appearance of electron neutrinos $[3,4]$ and the measurement of mixing angle $\theta_{13}$ [5] were obtained. Nevertheless, information about leptonic $\mathrm{CP}$ phase $\delta_{C P}$ and the neutrino mass hierarchy are still missing. On top of that the nature of neutrino (Dirac or Majorana particle) and the absolute mass scale are very open issues. In addition to these fundamental problems there are several experimental signals which might hint on the existence of sterile neutrinos. This brief review covers the recent progress in study of neutrino oscillations and measurements of the absolute scale of the neutrino mass.

\section{Measurements of oscillation parameters}

\subsection{Long baseline accelerator experiments $\mathrm{T} 2 \mathrm{~K}$ and $\mathrm{NO} v \mathrm{~A}$}

Two long baseline accelerator experiments, T2K in Japan and NOvA in the US, are now taking data. Both experiments use the so-called off-axis neutrino beam, which means that the beam axis is directed a few miliradians away from the far detectors. Thanks to the kinematics of pion decay, such configurations allow to obtain a quasi-monochromatic beam with the neutrino peak energy tuned to the oscillation maximum for a given baseline. The T2K (Tokaito-Kamioka) experiment uses the neutrino beam peaked at $0.6 \mathrm{GeV}$ directed from J-PARC toward the Super-Kamiokande detector $295 \mathrm{~km}$ away. T2K collects data since 2010 and recently released the result on the search for $\mathrm{CP}$ violation in neutrino oscillations based on $14.9 \times 10^{20}$ protons on target (POT) for the neutrino mode and $11.2 \times 10^{20}$ POT for antineutrino

\footnotetext{
*e-mail: kudenko@inr.ru
} 
mode. In total, $75 \mathrm{CCQE} v_{e}, 15 \mathrm{CC} 1 \pi$, and $9 \mathrm{CCQE} \bar{v}_{e}$ events were detected. Using the value of $\theta_{13}$ from reactor experiments $\mathrm{T} 2 \mathrm{~K}$ is able to constrain $\delta_{C P}$. T2K confidence intervals obtained for $\delta_{C P}$ are shown in Fig. 1. The vertical lines with hatching (black for normal,

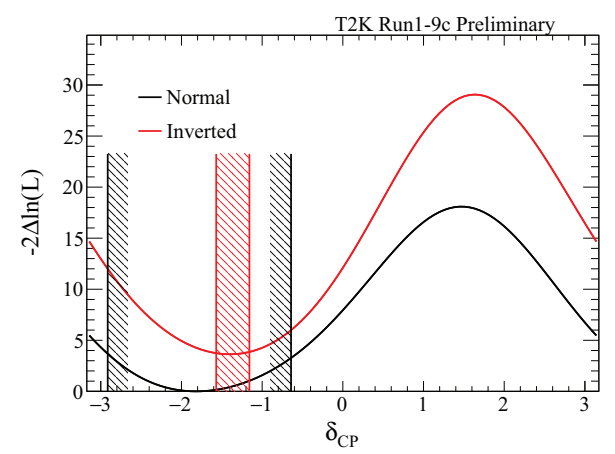

Figure 1. $-2 \Delta \ln (\mathrm{L})$ as a function of $\delta_{\mathrm{CP}}$ for the normal (black) and inverted (red) mass hierarchy using reactor constraint on $\theta_{13}$. The vertical lines show the corresponding allowed $2 \sigma$ confidence intervals.

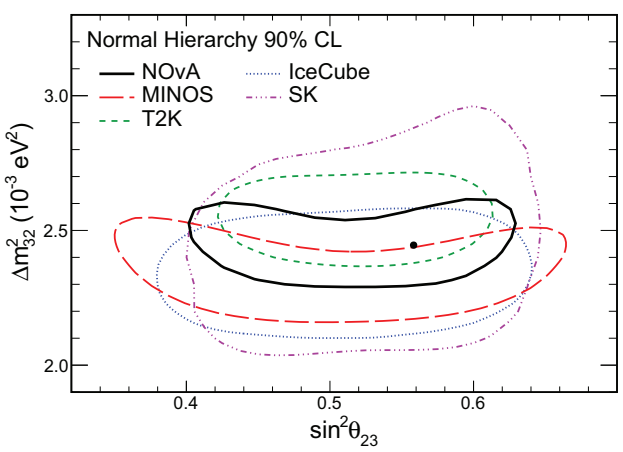

Figure 2. Two-dimensional $90 \%$ confidence level contours for $\Delta m_{32}^{2}$ vs $\sin ^{2} \theta_{23}$ of various experiments [6]. The black point is the best-fit value of $\mathrm{NO} v \mathrm{~A}$.

red for inverted mass hierarchy) limit the allowed $2 \sigma$ intervals for $\delta_{C P}$. As seen from this figure, $\mathrm{CP}$ conservation $\left(\delta_{C P}=0\right.$ or $\pi$ ) is excluded at $95 \%$ confidence level. The best fit value is $\delta_{C P}=-1.6 \mathrm{rad}$ for the normal mass hierarchy. This value is close to the maximal $\mathrm{CP}$ violation.

The NO $v$ A experiment has a baseline of $810 \mathrm{~km}$ and uses the neutrino beam with the peak energy about $2 \mathrm{GeV}$ around the $v_{\mu} \rightarrow v_{e}$ oscillation maximum. The experiment uses a 14-kt liquid scintillator far neutrino detector in Ash River, Minnesota, to detect the oscillated muon neutrino beam produced at Fermilab. The result for $v_{\mu}$ disappearance from an exposure of $8.85 \times 10^{20}$ POT and normal mass hierarchy is shown in Fig. 2. 90\% confidence level contours for these parameters in the normal mass hierarchy for T2K, MINOS, IceCube, and SuperKamiokande are also shown. All of the experiments have results consistent with maximal mixing. NOvA disfavors the inverted mass hierarchy at the $95 \%$ confidence level. It should be noted that both, T2K and NOvA have good chances to exclude $\mathrm{CP}$ conservation with a significance of $>3 \sigma$.

\section{Future long baseline projects}

A rich experimental program is under preparation to answer the fundamental questions in neutrino physics. Is there leptonic CP violating or not? What is the neutrino mass hierarchy? What is the value of $\theta_{23}$ ? The next generation of long baseline accelerator experiments, DUNE, T2HK and the reactor experiment JUNO have real chances to discover CP violation in neutrino oscillations and determine the neutrino mass hierarchy.

$J U N O$. The long baseline reactor experiment JUNO (Jiangmen Underground Neutrino Observatory) [7] has two main goals: the determination of the neutrino mass hierarchy and the precise measurement of oscillation parameters $\Delta m_{21}^{2}, \Delta m_{31}^{2}$, and $\sin ^{2} \theta_{12}$. The JUNO detector, a $20 \mathrm{kt}$ spherical unsegmented liquid scintillator detector, will be located at a distance of 53 $\mathrm{km}$ from the Yangjiang and Taishan nuclear power plants. The expected spectrum (dashed line shows the non-oscillating case) for a detector with a baseline of about $50 \mathrm{~km}$ is shown in 
Fig 3. There is a small ripple in the neutrino flux as a function of $\mathrm{L} / \mathrm{E}$ that depends on the mass

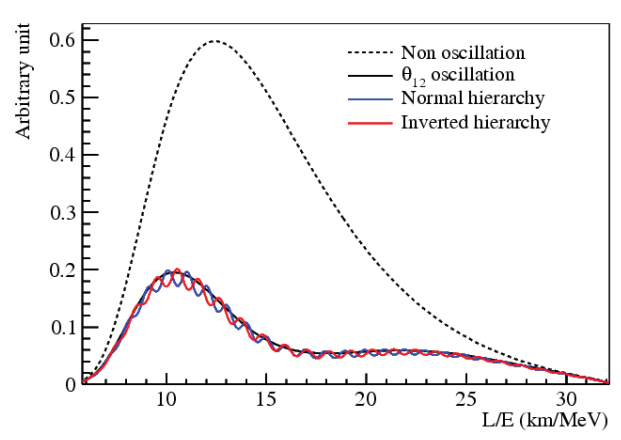

Figure 3. The shape of the electron antineutrino flux as a function of $L / E$ for different neutrino mass hierarchies for the JUNO experiment [7]. The figure represents the product of the neutrino flux times the interaction cross section times the survival probability.

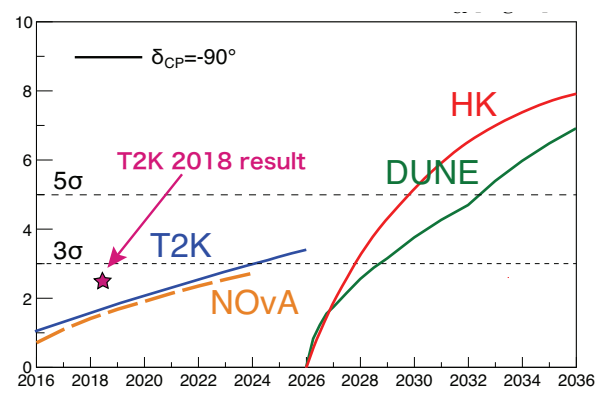

Figure 4. The expected significance of measurement of $\mathrm{CP}$ violation in HyperKamiokande (denoted as HK) and DUNE as a function of running time. The normal mass hierarchy, $\delta_{\mathrm{CP}}=-\pi / 2$, are assumed. $\mathrm{T} 2 \mathrm{~K}$ and $\mathrm{NO} v \mathrm{~A}$ experiments are also shown.

hierarchy. This gives JUNO good sensitivity for determining the neutrino mass hierarchy. To discriminate between the neutrino hierarchies at $\mathrm{a} \geq 3 \sigma$ level, the energy resolution of this detector is required to be $3 \% / \sqrt{E(\mathrm{MeV})}$ and the absolute energy scale should be calibrated with a precision of about $1 \%$. It is expected that after 6 years of data taking JUNO can distinguish between the true and wrong hierarchy hypothesis at a significance level of about $4 \sigma$. JUNO is under construction and plans to begin operation in 2021.

DUNE. The main scientific goals of the Deep Underground Neutrino Experiment (DUNE) [8] are the sensitive test of CP violation in the leptonic sector, determination the neutrino mass hierarchy, and precise measurements of neutrino oscillation parameters. The proposed liquid Argon far neutrino detector will be built deep underground, at a depth of about $1500 \mathrm{~m}$, in the Sanford Underground Research Facility (South Dakota, USA), about $1300 \mathrm{~km}$ from Fermilab where a high intensity wide band on-axis neutrino beam with neutrino energies of $1-6 \mathrm{GeV}$ will be formed. This neutrino beam will cover the first and the second oscillation maxima which correspond to the neutrino energy of $2.5 \mathrm{GeV}$ and $0.8 \mathrm{GeV}$, respectively. The far detector will consist of four cryostats instrumented with liquid Argon Time Projection Chambers with a fiducial mass of $40 \mathrm{kt}$. DUNE plans to begin data taking with the first 10 kton module in 2027 and the full configuration will be ready by 2029 .

Hyper-Kamiokande. This project will be focused on a sensitive measurement of CP violation in neutrino oscillations, on a search for proton decay and study of solar, atmospherics and astrophysical neutrinos [9]. A gigantic water Cherenkov Hyper-Kamiokande detector equipped with newly developed high efficiency and high-resolution PMTs will serve as a far detector in T2HK experiment which will use neutrino and antineutrino beams produced at J-PARC upgraded to the power of $\sim 1.3 \mathrm{MW}$. The baseline design includes one $260 \mathrm{kt}$ Cherenkov detector at a distance of $295 \mathrm{~km}$ from J-PARC. The inner detector region of the tank is viewed by 40,000 PMTs that provides a $40 \%$ photo-cathode coverage. As in T2K, the $2.5^{\circ}$ off-axis beam tuned to the first oscillation maximum will be used. The expected significance of measurement of CP violation in Hyper-Kamiokande and DUNE as a function of running time, for the normal mass hierarchy and $\delta_{\mathrm{CP}}=-\pi / 2$, is shown in Fig. 4 . The estimated sensitivities of $\mathrm{T} 2 \mathrm{~K}$ and $\mathrm{NO} v \mathrm{~A}$ are also shown. Both, Hyper-Kamiokande 
and DUNE, will be able to detect CP violation with the sensitivity of 7-8 $\sigma$ in case of its maximum violation.

\section{Search for sterile neutrinos}

Test of the LSND/MiniBooNe anomaly. There are a few experimental anomalies in study of neutrino oscillations which can be interpreted as hints of the existence of sterile neutrinos. A few new results on searches for sterile neutrinos have been obtained recently. The LSND/MiniBooNe anomaly was tested using the combined constraints derived from a search for electron neutrino disappearance at the Daya Bay and Bugey-3 reactor experiments and from a search for muon neutrino disappearance at the MINOS experiment [10]. This result is shown in Fig. 5. As seen in this figure, the sterile neutrino mixing phase space allowed

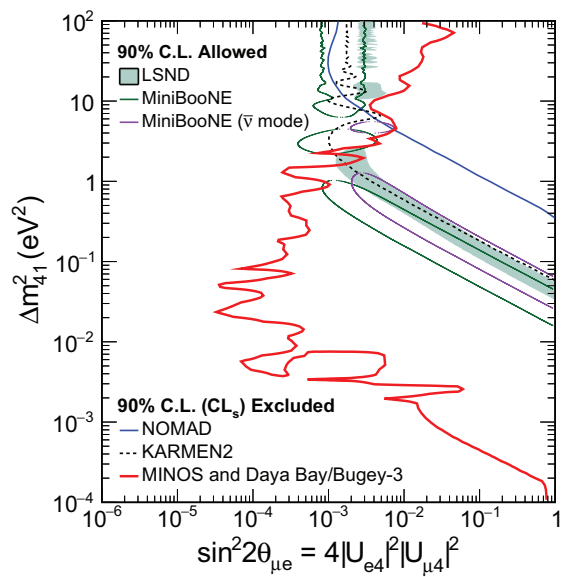

Figure 5. Daya Bay, MINOS and Bugey-3 combined 90\% CL limit on $\sin ^{2} 2 \theta_{\mu e}$ compared to the LSND and MiniBooNe 90\% CL allowed regions. The region to the right of the red contour is excluded.

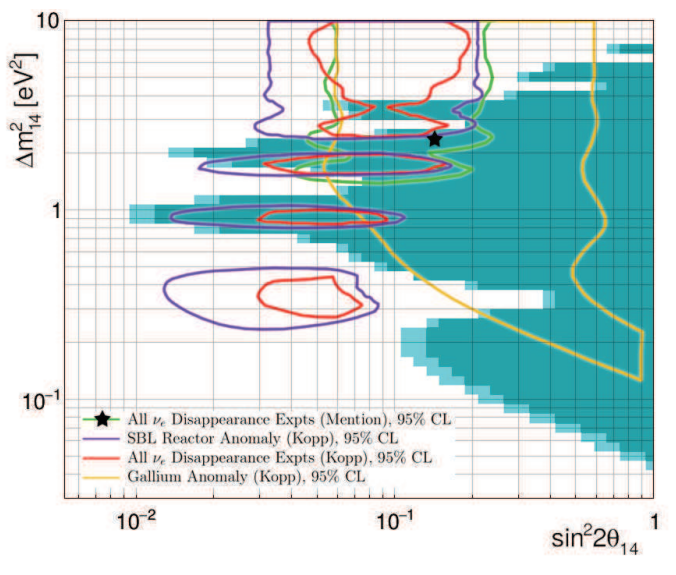

Figure 6. Exclusion contour obtained in the DANSS experiment [13]. The shaded areas show the $90 \%$ CL (cyan) and 95\% CL (dark cyan) excluded regions. Curves show the allowed regions from reactor disappearance experiments. The star shows the best-fit point from the reactor and gallium anomalies.

by the LSND and MiniBooNe experiments are excluded. There is the strong tension between appearance results from LSND and MiniBooNe and null results from disappearance searches. Test of the reactor anomaly. New constraints on $\bar{v}_{e}$ disappearance into sterile neutrinos were recently obtained in reactor experiments NEOS [11], Neutrino-4 [12], DANSS [13], PROSPECT [14], and STEREO [15]. Results obtained in these experiments are independent from neutrino flux predictions and insensitive to the predicted spectrum shape. The exclusion contour obtained in the DANSS experiment is shown in Fig. 6.

\section{Direct neutrino mass measurements}

The absolute scale of neutrino masses can be obtained directly in a model-independent way by measurements of the electron spectrum in the tritium beta decay. In this case the observable is incoherent sum of three neutrino masses: $m_{\beta}^{2}=\sum_{i}\left|U_{e i}\right|^{2} m_{i}^{2}$. The KATRIN experiment aims at measuring the effective electron antineutrino mass with a $0.2 \mathrm{eV}$ (90\% CL) sensitivity 
by analyzing the beta-decay spectrum of molecular tritium near its endpoint. The $70 \mathrm{~m}$-long KATRIN setup consists of a windowless gaseous source, differential and cryogenic pumps for tritium retention, and a main spectrometer acting as a high-pass filter for electrons collimated towards the silicon focal plane detector. In 2018, the set-up was successfully tested and took its commissioning data using a gaseous tritium source. The first neutrino mass runs are expected in 2019. The expected sensitivity of KATRIN to the neutrino mass as a function of running time is shown in Fig. 7.

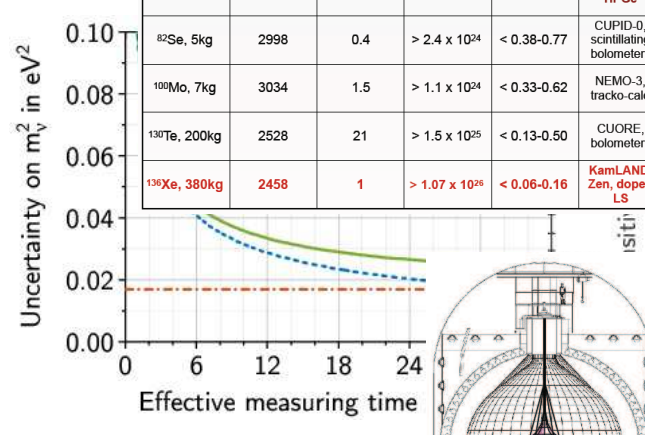

Figure 7. Sensitivity of the KATRIN experiment as a function of the running time. Left vertical axis: $1 \sigma$ statistical, systematic and total uncertainties of $m_{v}^{2}$. Right vertical axis: sensitivity (90\% CL) to $m_{v}[16]$.

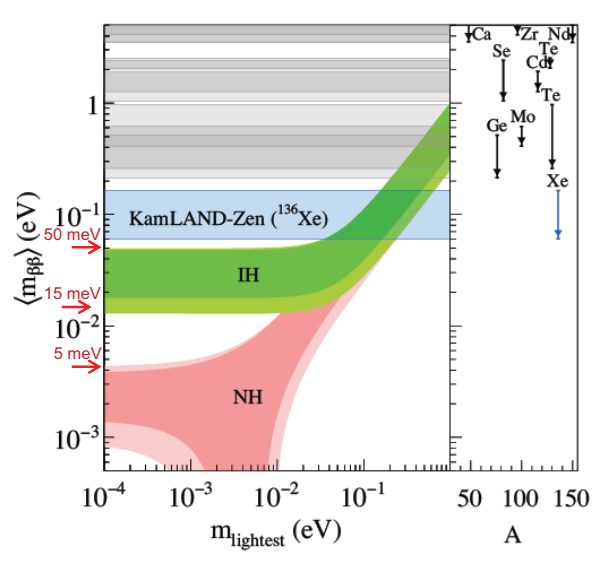

Figure 8. Upper limits (90\% CL) on the effective mass $<m_{\beta \beta}>$ from recent $0 v 2 \beta$ search experiments and the allowed regions for the normal mass hierarchy $(\mathrm{NH})$, the inverted mass hierarchy $(\mathrm{IH})$ and the quasi-degenerated regions provided by the neutrino oscillation experiments.

\section{Search for neutrinoless double beta decay}

Neutrinoless double beta decay $(0 v 2 \beta)$ is forbidden in the Standard Model. Its discovery would be a major step in neutrino physics since it would directly confirm lepton number violation and the Majorana nature of neutrinos [17]. Many experiments are now looking for this decay using different techniques and technologies. The main approach is to search for a two electron signal with a monoenergetic peak as there are no antineutrinos emitted in the decay. The most critical consideration is the potential sources of backgrounds. An irreducible background is the $2 v \beta \beta$-decay electrons. Searches for $0 v 2 \beta$ decay are carried out in a number of experiments with different nuclei. The results of the most sensitive to the $0 v 2 \beta$ decay experiments are presented below.

The GERDA experiment searches the $0 v 2 \beta$ decay of ${ }^{76} \mathrm{Ge}$ using bare Ge detectors with an enriched ${ }^{76} \mathrm{Ge}$ fraction in liquid argon which cools the detectors and shields them from external radiation. GERDA is the first background free experiment for all expected exposure. No signal was observed and a new 90\% CL lower limit for the half-life of $8 \times 10^{26} \mathrm{yr}$ is obtained based on the total exposure of $471.1 \pm 8.5 \mathrm{~mol} \cdot \mathrm{yr}$ of ${ }^{76} \mathrm{Ge}$ in the active volume of the detectors [18]. The EXO-200 collaboration employs a liquid Time Projection Chamber based on Xe. With an energy resolution of $\sigma \sim 1.23 \% / E$ and about $75 \mathrm{~kg}$ of Xe in the fiducial 
volume, EXO-200 reached a limit of $T_{1 / 2}^{0 v}>1.8 \times 10^{25} \mathrm{yr}$ with the upgraded detector [19]. The KamLAND-Zen experiment reached the most impressive limit on the half-life of $0 v 2 \beta$. This experiment, located in the Kamioka mine (Japan) exploits the KamLAND facility, in which 1000 tons of liquid scintillator were deployed in a $13 \mathrm{~m}$ diameter balloon. The KamLAND detector was upgraded for $0 v 2 \beta$ searches, with the insertion of a mini-ballon containing liquid scintillator loaded with Xe $90.6 \%$ enriched in ${ }^{136} \mathrm{Xe}$. The combined analysis of phase-I and phase-II data accumulated by KamLAND-Zen provides the most competitive limit on the $0 v 2 \beta$ half-life: $T_{1 / 2}^{0 v}>1.07 \times 10^{26} \mathrm{yr}$, corresponding to $m_{\beta \beta}<61-165 \mathrm{meV}$ [20]. Figure 8 shows the limits on the effective neutrino mass $\left\langle m_{\beta \beta}>\right.$ as a function of the lightest neutrino mass. The stringent constraint by KamLAND-Zen excludes most of the quasi degenerate region and approaches the inverted mass hierarchy region.

\section{Conclusion}

The discovery of neutrino oscillations opened a window to new physics that is being intensively studied. A very broad research program is now focused on the search for CP violation in leptonic sector and determination of the neutrino mass hierarchy. Anomalies that may point to existence of sterile neutrinos are being addressed by many experiments. Direct measurement of the absolute mass scale is underway and the sensitivity of neutrinoless double beta decay experiments becomes sufficient to test the inverted mass hierarchy.

\section{Acknowledgments}

This work was supported in part by the Russian Science Foundation grant \# 19-12-00325.

\section{References}

[1] T.Kajita, Rev. Mod. Phys. 88030501 (2016).

[2] A.B.McDonald, Rev. Mod. Phys. 88030502 (2016).

[3] K.Abe et al., Phys. Rev. Lett. 107041801 (2011).

[4] K.Abe et al., Phys. Rev. Lett. 112061802 (2014).

[5] F.P.An et al., Phys. Rev. Lett. 108171803 (2012).

[6] M.Acero et al., Phys.Rev. D98 032012 (2018).

[7] F.An et al., J.Physics G 43030401 (2016).

[8] R.Acciarri et al., arXiv:1512.06148 [physics.ins-det].

[9] K.Abe et al., arXiv:1805.04163 [physics.ins-det].

[10] P.Adamson et al., Phys.Rev.Lett. 117151801 (2016).

[11] Y.J.Ko et al., Phys. Rev. Lett. 118121802 (2017).

[12] A.Serebrov et al., JETP Lett. 109209 (2019).

[13] I.Alexeev et al., Phys. Lett. B787 56 (2018).

[14] J.Ashenfelter et al., Phys.Rev.Lett. 121251802 (2018).

[15] H.Almazan et al., Phys. Rev. Lett. 121161801 (2018).

[16] M.Kleesiek et al., Eur.Phys.J. C79 204 (2019).

[17] J.Schechter, J.W.F.Valle, Phys. Rev. D25 2951 (1982).

[18] M.Agostini et al., Phys.Rev.Lett. 120132503 (2018).

[19] J.B. Albert et al., Phys.Rev.Lett. 120072701 (2018).

[20] A.Gando et al., Phys.Rev.Lett. 117, 082503 (2016). 